\title{
SUBJECT INDEX VOLUME 16
}

\section{A}

ACTH, cocaine and, 399

Addiction, cocaine, 77

ADHD. See Attention deficit/hyperactivity disorder (ADHD)

$\alpha_{2}$-Adrenoceptor antagonist, gender and, 298

Adrenocorticotrophic hormone (ACTH). See $\mathrm{ACTH}$

$\beta$-Adrenoreceptors, alcoholism, panic disorder, and, 69

Affective disorders, lithium and, 408

Aggression, platelet 5- $\mathrm{HT}_{2 \mathrm{~A}}$ receptors and, 211

Aging, somatostatin effects on sleep, 339

Alcoholism, $\beta$-adrenoreceptors in panic disorder and, 69

Alpha-ethyltryptamine (AET), effects on prepulse inhibition (PPI) and startle habituation, 246

Alprazolam

CYP2C19 status and, 8

S-mephenytoin 4-hydroxylation and, 8 smoking and, 8

Alzheimer's disease, thiopental and, 269

Amino acids, effects on tardive dyskinesia with schizophrenia, 136

d-Amphetamine, opiate-dopamine interactions, 147

Amphetamines, neonatal hippocampal lesion-induced behavioral hyperresponsiveness and, 259

Anticonvulsants, valproic acid, 238

Antipsychotic drugs, effects on dopamine $\mathrm{D}_{2}$ and $5-\mathrm{HT}_{2}$ receptors, 1

AP-1, valproic acid and, 238

Attention, seligiline in attention deficit/ hyperactivity disorder (ADHD) adults, 276

Attention deficit/hyperactivity disorder (ADHD), selegiline in adults, 276

Atypical antipsychotic drugs, olanzapine effects on dopamine $\mathrm{D}_{2}$ and $5-\mathrm{HT}_{2}$ receptors, 1

\section{B}

Beta activity, thiopental in dementia and, 269

Bipolar disorder

lithium and, 325

valproic acid and, 238

Blood platelets, serotonin receptors and, 285

Blood pressure, serotonin receptors and, 285

Body mass index, serotonin receptors and 285

Body weight, serotonin receptors and, 285

Brain

lithium and carbamazepine effects on rat, 25

ventricular brain ratio, schizophrenia in drug-naive patients and, 51

\section{C}

c-fos, binding assays, 238

c-jun, binding assays, 238

cAMP responsive element binding protein (CREB), valproic acid and, 238

Cannabinoids, prefrontal cortica] catecholaminergic utilization, spatial working memory, and, 426

Carbamazepine, thyroid hormone metabolism and, 25

Catalepsy, atypical neuroleptics and, 93, 111, $114,116,123$

Catecholamines, selective attention and, 202

CCK, social phobia and, 229

Cell membrane, lithium in bipolar disorder and, 325

Cerebrospinal fluid (CSF), levels in transforming growth factors with schizophrenia, 83

Chemical models, social phobia and, 229

$\mathrm{m}$-Chlorophenylpiperazine (m-CCP), premenstrual dysphoric disorder (PMDD) and, 346

Cholecystokinin (CCK), social phobia and, 229

Cholinergic, lithium and basal and stimulated $c$-fos mRNA expression in rat brain, 408

Clonidine, selective attention and, 202

Clozapine

dopamine $D_{2}$ and $D_{4}$ receptors and, 93 , $111,114,116,123$

prefrontal cortical cognitive defects and, 433

Cocaine

corticosterone blockade and, 156

gender and cocaine-induced HPA axis activation, 399

opiate-dopamine interactions, 147

sensitization, 77

Cognition

cannabinoids and, 426

cholinergic, dopaminergic, and serotonergic blockade effects, 15

clozapine and, 433

Contraceptives, oral, serotonin receptors and, 285

Cortical development, schizophrenia and, 385

Corticosterone, cocaine and, 156

Cortisol, D-cycloserine and, 317

CREB, valproic acid and, 238

D-Cycloserine, behavioral and neuroendocrine effects, 317

CYP2C19 status, alprazolam and, 8

Cytochrome P450

alprazolam and, 8

serotonin receptors and, 285

\section{D}

Deiodinases, lithium and carbamazepine effects, 25
Delayed alteration, cannabinoids and, 426

Delayed response task, clozapine and, 433

Depression

diabetes and, 183

major, selective serotonin reuptake inhibitors (SSRI) effects on $5-\mathrm{HT}_{1 \mathrm{~A}}$ agonists, 333

serotonin receptors in suicide victims, 162

tryptophan depletion and, 294

valproic acid and, 238

Diabetes, effects on dopamine and serotonin receptors, 183

Dopamine

clozapine and, 433

neuronal circuits and, 385

psychosis, 191

schizophrenia and, 61

Dopamine $D_{1}$ receptors, neonatal hippocampal lesions and, 259

Dopamine $\mathrm{D}_{2}$ receptors

naloxone-precipitated withdrawal with opiate-dependence and, 174

neonatal hippocampal lesions and, 259

neuronal circuits and, 375

olanzapine effects on $5-\mathrm{HT}_{2}$ and, 1

Dopamine $\mathrm{D}_{3}$ receptors, neuronal circuits and, 375

Dopamine $\mathrm{D}_{4}$ receptors

atypical neuroleptics and, 93, 111, 114, 116,123

neuronal circuits and, 375

Dopamine receptors, streptozotocininduced diabetes effects, 183

Dopaminergic, lithium and basal and stimulated $c$-fos mRNA exp ssion in
rat brain, 408

Droperidol, selective attention and, 202

Drug-naive patients, schizophrenia in, 51

Drug therapy, bipolar disorder and, 325

$\mathbf{E}$

EEG, thiopental in dementia and, 269

EEG sleep, schizophrenia in drug-naive patients and, 51

ELISA, transforming growth factors and schizophrenia, 83

Event-related potential (ERP), selective attention and, 202

Eye movement, risperidone and haloperidol effects, 217

$\mathbf{F}$

Females, cocaine-induced HPA axis activation and, 399

First-episode schizophrenia, mchlorophenyl-piperazine vs. methylphenidate, 61

${ }^{18}$ F-Fluoro-deoxyglucose (FDG), gender and idazoxon effects, 298

[F-18]-Fluorodeoxyglucose (FDG) psilocybin model of psychosis, 357

NEUROPSYCHOPHARMACOLOGY 1997-VOL. 16, NO. 6

(C) 1997 American College of Neuropsychopharmacology

Published by Elsevier Science Inc. 
Fluoxetine

alpha-ethyltryptamine (AET) effects on

startle habituation and, 246

effects on extracellular norepinephrine in hippocampus, 419

premenstrual dysphoric disorder (PMDD) and, 346

Follicular phase, fluoxetine and, 346 $c$-fos mRNA expression, lithium and, 408

Fuller, Ray, in memoriam, 256

\section{G}

Gender, cocaine-induced HPA axis activation and, 399

GHRH, somatostatin and sleep effects in elderly and, 339

Growth hormone-releasing hormone (GHRH). See GHRH

\section{$\mathbf{H}$}

Habituation, alpha-ethyltryptamine (AET) and, 246

Haloperidol

cognitive and behavioral effects, 15

effects on dopamine and serotonin receptors, 183

saccadic eye movements and, 217

Heterogeneity, dopamine psychosis, 191

Hippocampus

dopamine receptors and, 259

serotonin receptors in suicide victims with depression and, 162

Homovanillic acid, psychosis, 191

HPA axis activation, gender and cocaineinduced, 399

5-HT agonists, alpha-ethyltryptamine (AET) effects on startle habituation and, 246

$5-\mathrm{HT}_{1 \mathrm{~A}}$ agonists, selective serotonin reuptake inhibitors (SSRI) effects, 333

$5-\mathrm{H}_{2}$ receptors olanzapine effects on dopamine $\mathrm{D}_{2}$ and, 1 psilocybin model of psychosis, 357

Humans

positron emission tomography study of 5- $\mathrm{HT}_{2}$ and dopamine $\mathrm{D}_{2}$ receptor occupancy, 1

psilocybin model of psychosis, 357

Hyperactivity, seligiline in attention deficit/ hyperactivity disorder (ADHD) adults, 276

Hypothalamo-pituitary-adrenal (HPA) axis. See HPA axis

Idazoxan, gender and responses to $\alpha_{2}$ adrenoceptor blockade, 298

Imidazoline, gender and responses to $\alpha_{2}-$ adrenoceptor blockade, 298

In memoriam, Winokur, George, 438

In vivo microdialysis, fluoxetine effects on extracellular norepinephrine in hippocampus, 419

Ion transport, lithium in bipolar disorder and, 325

\section{L}

Latent inhibition, neuroleptic-induced enhancement, 42

LH, D-cycloserine and, 317
Lithium

basal and stimulated $c$-fos mRNA expression in rat brain and, 408 bipolar disorder and, 325

thyroid hormone metabolism and, 25

Locomotion

corticosterone blockade effects on cocaine, 156

dopaminergic agonists and antagonists and, 259

opiate-dopamine interactions and, 147

Locus coeruleus, fluoxetine effects on extracellular norepinephrine, 419

Luteal phase, fluoxetine and, 346

Luteinizing hormone (LH). See LH

Lysergic acid diethylamide, serotonin receptors and, 285

\section{$\mathbf{M}$}

M-chlorophenyl-piperazine, schizophrenia and methylphenidate vs., 61

Major depression, selective serotonin reuptake inhibitors (SSRI) effects on 5- $\mathrm{HT}_{1 \text { A }}$ agonists, 333

Mania, valproic acid and, 238

MAOI, attention deficit/hyperactivity disorder (ADHD) and, 276

Memory, cannabinoids and, 426

Menstrual cycle, serotonin receptors and, 285

Metabolism, alprazolam and, 8

Metergoline, cognitive and behavioral effects, 15

N-Methyl-D-Aspartate (NMDA) receptors. See NMDA receptors

3,4-Methylenedioxy-Nmethylamphetamine (MDMA), alphaethyltryptamine (AET) vs., 246

Methylphenidate, schizophrenia and m-chlorophenyl-piperazine vs., 61

Metyrapone, corticosterone blockade effects on cocaine and, 156

Microdialysis, in vivo, fluoxetine effects on

extracellular norepinephrine in hippocampus, 419

Monoamine metabolites, attention deficit/ hyperactivity disorder (ADHD) and, 276

Monoamines, prefrontal cortical catecholaminergic utilization, spatial working memory, and, 426

Morphine, opiate-dopamine interactions, 147

\section{$\mathbf{N}$}

Naloxone, dopamine $\mathrm{D}_{2}$ receptors in opiatedependence and, 174

Neonatal lesions, dopamine receptors and, 259

Neuroleptic response, homovanillic acid psychosis and, 191

Neuroleptics

dopamine $\mathrm{D}_{2}$ and $\mathrm{D}_{4}$ receptors and, 93 $111,114,116,123$

latent inhibition and, 42

Neuronal circuits, schizophrenia and, 375 , 385

Neurotoxins, neonatal hippocampal lesions and, 259

NMDA receptors, behavioral and neuroendocrine effects, 317

Norepinephrine, fluoxetine effects on extracellular, 419
Nucleus accumbens, opiate-dopamine interactions and, 147

O

Obsessive compulsive disorder (OCD), alpha-ethyltryptamine (AET) and, 246

Olanzapine, effects on dopamine $\mathrm{D}_{2}$ and $5-\mathrm{HT}_{2}$ receptors,

$\mathbf{P}$

P3, catecholamine transmission and, 202

Panic, pentagastrin and, 229

Panic disorder, $\beta$-adrenoreceptors in alcoholism and, 69

Parkinsonism, atypical neuroleptics and, 93, $111,114,116,123$

Pentagastrin, social phobia and, 229

PET. See Positron emission tomography (PET)

Pharmacology

M-chlorophenyl-piperazine, 61

methylphenidate, 61

Phenylalanine, effects on tardive dyskinesia with schizophrenia, 136

Pindolol, effects on 5- $\mathrm{HT}_{1 \mathrm{~A}}$ agonists for antidepressant response, 333

Plasma monoamines, attention deficit/ hyperactivity disorder (ADHD) and, 276

Platelet $5-\mathrm{HT}_{2} \mathrm{~A}$ receptors, effects with personality disorder, 211

Positron emission tomography (PET)

dopamine $D_{2}$ receptors in opiatedependence and, 174

gender and $\alpha_{2}$-adrenoceptor blockade, 298

$5-\mathrm{HT}_{2}$ and dopamine $\mathrm{D}_{2}$ receptor occupancy, 1

psilocybin model of psychosis, 357

Prefrontal cortex

cannabinoids and, 426

clozapine and, 433

schizophrenia and, 385

serotonin receptors in suicide victims with depression and, 162

Prepulse inhibition (PPI), alphaethyltryptamine (AET) and, 246

Probes, M-chlorophenyl-piperazine vs. methylphenidate in schizophrenia, 61

Processing negativity, catecholamine transmission and, 202

Psilocybin, psychosis model, 357

Psychomimetics, prefrontal cortical catecholaminergic utilization, spatial working memory, and, 426

Psychomotor effects, corticosterone blockade effects on cocaine, 156

Psychopathology, psilocybin model of psychosis, 357

Psychosis

cocaine, 77

dopamine, 191

psilocybin model, 357

Pyramidal neurons, schizophrenia and, 385

$\mathbf{R}$

Rapid eye movement latency, schizophrenia Rats in drug-naive patients and, 51

lithium and basal and stimulated $c$-fos mRNA expression, 408

lithium and carbamazepine effects, 25 
neuroleptic-induced enhancement of latent inhibition, 42

Receptors, serotonin, binding variability, 285

Relapse

cocaine addiction, 77

former depressed patients and tryptophan depletion, 294

REM sleep, elderly and somatostatin, 339

Risperidone, saccadic eye movements and, 217

\section{S}

S-mephenytoin 4-hydroxylation, alprazolam and, 8

Saccades, risperidone and haloperidol effects, 217

Schizophrenia

clozapine and, 433

D-cycloserine and, 317

dopamine psychosis and, 191

dopamine receptors and, 375,385

EEG sleep in drug-naive patients, 51

$\mathrm{m}$-chlorophenyl-piperazine vs.

methylphenidate, 61

phenylalanine effects with tardive dyskinesia and, 136

premenstrual dysphoric disorder (PMDD) and, 346

psilocybin model of psychosis, 357

transforming growth factors and, 83

Scopolamine, cognitive and behavioral effects, 15

Seasons, serotonin receptors and, 285

Selective attention, catecholamine transmission and, 202

Selective serotonin reuptake inhibitors (SSRI), effects on $5-\mathrm{HT}_{1 \mathrm{~A}}$ agonists for antidepressant response, 333

Seligiline, attention deficit/hyperactivity disorder (ADHD) adults and, 276
Sensitization

cocaine, 77

opiate-dopamine interactions, 147

Serotonin

aggression in personality disorder and, 211

alpha-ethyltryptamine (AET) effects on startle habituation and, 246

schizophrenia and, 61

tryptophan depletion in former depressed patients and, 294

Serotonin receptors

streptozotocin-induced diabetes effects 183

suicide victims with depression and, 162

Serotonin 2 receptors, atypical neuroleptics and, 93, 111, 114, 116, 123

Sex characteristics, $\alpha_{2}$-adrenoceptor blockade and, 298

Side effects, risperidone and haloperidol, 217

Signal transduction, lithium and basal and stimulated $c$-fos mRNA expression in rat brain, 408

Sleep

elderly and somatostatin, 339

slow wave, schizophrenia in drug-naive patients and, 51

Sleep endocrinology, elderly and somatostatin, 339

Slow wave sleep, schizophrenia in drugnaive patients and, 51

Smoking, alprazolam and, 8

Social interaction, pentagastrin and, 229

Somatostatin, sleep effects in elderly, 339

Startle habituation, alpha-ethyltryptamine (AET) and, 246

Streptozotocin,-induced diabetes, effects on dopamine and serotonin receptors, 183

Stress

clozapine and, 433 fluoxetine effects on extracellular norepinephrine in hippocampus and, 419

lithium and basal and stimulated $c$-fos mRNA expression in rat brain, 408

Suicide, serotonin receptors and, 162

Switching, neuroleptics, 42

\section{$\mathbf{T}$}

Tardive dyskinesia

diabetes and, 183

phenylalanine in schizophrenia with, 136

Thiopental, Alzheimer's type dementia and, 269

Thyroid hormones, lithium and carbamazepine effects, 25

Transcription factors, valproic acid and, 238

Transforming growth factors, schizophrenia and, 83

Tricyclic antidepressants, effects on $5-\mathrm{HT}_{1 \mathrm{~A}}$ agonists for antidepressant response, 333

Tryptophan depletion, former depressed patients and, 294

V

Valproic acid, AP-1 transcription factor DNA binding activity and, 238

Vascular dementia, thiopental and, 269

Ventricular brain ratio, schizophrenia in drug-naive patients and, 51

\section{W}

Winokur, George, in memoriam, 438 Withdrawal, dopamine $D_{2}$ receptors in opiate-dependence and, 174 